\title{
Prenatal plasma concentrations of Perfluoroalkyl and polyfluoroalkyl substances and neuropsychological development in children at four years of age
}

Jinbo Niu ${ }^{1 \dagger}$, Hong Liang ${ }^{2 \dagger}$, Youping Tian², Wei Yuan², Hong Xiao ${ }^{3}$, Hui Hu${ }^{4}$, Xiaowei Sun ${ }^{2}$, Xiuxia Song ${ }^{2}$, Sheng Wen ${ }^{5}$, Li Yang ${ }^{6}$, Yanfeng Ren ${ }^{7 *}$ and Maohua Miao ${ }^{2}$

\begin{abstract}
Objective: Perfluoroalkyl and polyfluoroalkyl substances (PFASs) are persistent pollutants and have endocrine disruptive and neurotoxic effects. The association between maternal PFAS concentrations and neuropsychological development in children is inconclusive. The present study aimed to examine the effect of maternal PFAS concentrations on neuropsychological development in 4-years-old children.

Methods: We used data from Shanghai-Minhang Birth Cohort, which recruited pregnant women at 12-16 gestational weeks. Among 981 women having PFAS measurement, 533 mother-child pairs were included in the study. A total of eight PFASs were measured, including perfluorooctane sulfonate (PFOS), perfluorooctanoic acid (PFOA), perfluorohexane sulfonate (PFHXS), perfluorononanoic acid (PFNA), perfluorodecanoic acid (PFDA), perfluoroundecanoic acid (PFUdA), perfluorododecanoic acid (PFDoA), and perfluorotridecanoic acid (PFTrDA). When infants turned 4 years old, mothers were asked to complete the Ages and Stages Questionnaires ${ }^{\circledR}$ (ASQ) to assess neuropsychological development of their children. Poisson regression model with robust variance estimates was used to examine the association between maternal PFAS concentrations and each developmental subscale of the ASQ.

Results: Prenatal plasma concentrations of most PFASs tended to be associated with increased risk of development problem in personal-social skills, including PFHxS, PFOS, PFOA, PFNA, PFDA, and PDUdA, and the associations for PFNA and PFDA were significant (per natural log unit increase: $R_{\text {PFNA }}=1.92,95 \% \mathrm{Cl}: 1.21,3.05$; RR PFDA $=1.66,95 \%$ Cl: 1.17 , 2.37). In stratified analyses by child' sex, the consistent pattern of higher risk of developmental problems in personalsocial skills associated with most PFASs was mainly observed among girls (RRPFOS $=2.56,95 \%$ Cl: 1.20, 5.45; RRPFOA $=$ 9.00, 95\% Cl: 3.82, 21.21; RRPFNA $=3.11,95 \%$ Cl: $1.36,7.13 ; R_{\text {PFDA }}=2.20,95 \%$ Cl: $1.21,4.00 ; R_{\text {PFUdA }}=2.44,95 \%$ Cl: 1.14 , 5.20; RRPFDoA $=1.62,95 \% \mathrm{Cl}: 1.04,2.54)$. Boys with higher maternal PFOA concentrations had a decreased risk of developmental problems in gross motor skills $(\mathrm{RR}=0.47,95 \% \mathrm{Cl}: 0.25,0.89)$.

*Correspondence: renyf1978@163.com

${ }^{\dagger}$ Jinbo Niu and Hong Liang contributed equally to this work.

${ }^{7}$ Department of Health Statistics, School of Public Health and Management,

Weifang Medical University, 7166 Baotong west Road, Weifang 261053,

Shandong Province, China

Full list of author information is available at the end of the article

(c) The Author(s). 2019 Open Access This article is distributed under the terms of the Creative Commons Attribution 4.0 International License (http://creativecommons.org/licenses/by/4.0/), which permits unrestricted use, distribution, and reproduction in any medium, provided you give appropriate credit to the original author(s) and the source, provide a link to the Creative Commons license, and indicate if changes were made. The Creative Commons Public Domain Dedication waiver (http://creativecommons.org/publicdomain/zero/1.0/) applies to the data made available in this article, unless otherwise stated. 
(Continued from previous page)

Conclusion: Prenatal plasma PFAS concentrations were associated with neuropsychological development in girls at 4 years of age, mainly in the subset of personal-social skills.

Keywords: Perfluoroalkyl and polyfluoroalkyl substances, Neuropsychology, Age and stage questionnaire, Prenatal concentrations

\section{Introduction}

Perfluoroalkyl and polyfluoroalkyl substances (PFASs), a group of synthetic chemicals with hydrophobic (waterrepelling) and oleophobic (oil-repelling) properties, have been extensively used in many consumer products, including oil, stain, grease, and water-repellent coatings on carpet, textiles, leather, and paper [1]. PFASs are bioaccumulative and have a long elimination half-life of 29 years [2]. Thus, they have been detected in wildlife and humans worldwide [3]. Concerns about the health/toxic effects of PFASs, particularly for in-utero exposure, have been raised for decades [4].

In animal studies, maternal PFAS exposure during pregnancy was associated with somatic growth, e.g., birth weight and size [5]. Moreover, maternal PFAS exposure can induce alteration in neuropsychological development of fetuses and neonates. Deranged spontaneous behavior was observed in adult mice following perfluorooctane sulfonate (PFOS) and perfluorooctanoic acid (PFOA) exposure during neonatal period, manifesting in hyperactivity and irreversibly reduced habituation [6]. In addition, maternal PFOS or PFOA exposure during pregnancy can lead to decreased motor function and delayed learning in rat offspring [7].

The associations between maternal PFAS exposure and neuropsychological development in children have also been examined in human studies, but the findings are inconsistent [8-11]. A prospective birth cohort study in Japan showed an association between prenatal PFOA exposure and mental developmental in girls aged 6 months, but not in those aged 18 months [12]. The biopersistent organochlorines in diet and human fertility (INUENDO) cohort study reported that prenatal exposure to PFOS and PFOA may have adverse effects on children's neurobehavioral development, specifically in terms of hyperactive behavior [13]. However, in the Danish National Birth Cohort (DNBC) established between 1996 and 2002, maternal serum levels of PFOA and PFOS were not associated with behavioral and motor coordination problems in 7-year old children [10]. A nested case-control study from the DNBC suggested that prenatal PFAS exposure did not increase the risk of attention-deficit/hyperactivity disorder (ADHD) or autism in children [11]. In a 2005-2006 cohort study in the Mid-Ohio Valley, children in the highest quartile of maternal PFOA concentrations had higher intelligence quotient scores and decreased ADHD scores at ages 612 years compared with those in the lowest quartile [8].

Previous studies examining the associations between maternal PFAS exposure and child neurodevelopment focused on PFOS and PFOA. However, other commonly used PFAS compounds, such as perfluorohexane sulfonate (PFHxS), perfluorononanoic acid (PFNA), and perfluorodecanoic acid (PFDA), can be detected in more than $85 \%$ of individuals in China and in France $[14,15]$ and $50 \%$ of individuals in USA [16] and are less studied. Maternal concentrations of PFASs, including PFOS, PFOA PFHxS, PFNA, and PFDA, are much higher in the Shanghai area than in the United States, Europe and Asian countries [14]. In the present study, we used data from the Shanghai-Minhang Birth Cohort Study (SMBCS) to examine associations between maternal concentrations of eight PFASs and the neurodevelopment of 4-year-old children assessed by the Ages and Stages Questionnaires, 3rd edition ${ }^{\circ}$ (ASQ - 3).

\section{Methods}

\section{Participants}

The Shanghai-Minhang Birth Cohort was established between April and December 2012 [14, 17]. While attending routine antenatal examinations at the Maternal and Child Health Hospital of Minhang district in Shanghai, all pregnant women at 12-16 weeks of gestation was invited to participate in the study. Inclusion criteria included: being registered residents of Shanghai, having no history of chronic disease of the liver, kidney, or other organs, planning to deliver in this study hospital, and willingness to participate in specified interviews during pregnancy and after delivery.

In total, 1292 eligible pregnant women completed a structured questionnaire, among them, 981 provided a fasting blood sample at enrollment. Sixty-seven eligible pregnant women were excluded due to referral to other hospitals $(n=28)$, twin pregnancy $(n=8)$, and abortion or stillbirth $(n=31)$. The remaining 1225 women delivered singleton live births. Structured questionnaires were administered postnatally during home visits at 4 years of age to collect information on the child's physical and neuropsychological development. ASQ-3 included in the structured questionnaire was used to identify children at 
a risk for neuropsychological developmental delay. We obtained complete ASQ-3 assessment data from 661 participants at 4 years of age. The present study included 533 mother-infant pairs who had measurements of prenatal PFAS concentration and child neuropsychological development at 4 years of age.

\section{Exposure assessment}

Maternal blood samples were collected at enrollment. After separating plasma from whole blood, the plasma samples were stored at $-80^{\circ} \mathrm{C}$ until shipment using dry ice to the Center for Disease Control and Prevention in Hubei Province for quantitative analyses of 11 PFASs.

Eleven PFASs in each plasma sample were measured using high-performance liquid chromatography coupled with tandem mass spectrometry (Agilent Technologies Inc., USA). Detailed information on sample preparation, separation, quantification, quality control, and limit of detection (LOD) has been described previously [14]. The following eight PFASs with detection rates $\geq 90 \%$ were included in the present study for statistical analyses: PFHxS, PFOS, PFOA, PFNA, PFDA, perfluoroundecanoic acid (PFUdA), perfluorododecanoic acid (PFDoA), and perfluorotridecanoic acid (PFTrDA).

\section{Assessment of children's neuropsychological development}

ASQ-3, contains 30 items designed to assess infant neuropsychological development in children aged 1-60 months. It covers five developmental subscales: communication, gross motor function, fine motor function, problemsolving ability, and personal-social skills [18]. A detailed description of Chinese translation, training, and process of validation of ASQ-3 has been described elsewhere [19]. The Simplified Chinese version of ASQ-3 has good internal consistency (Cronbach's $\alpha=0.80$ ), high test-retest reliability (correlation coefficient $=0.8$ ), and high validity $($ sensitivity $=87.50 \%$, specificity $=84.48 \%)$ [19].

At home visits, parents or other caregivers were asked whether the child performs the described behavior based on three alternatives: "yes" (10 points), "sometimes" (5 points) and "not yet" (0 points). The score of each ASQ3 subscale was highly skewed, with few children scoring lower than 2 standard deviations (SDs) below the standardized mean. Thus, the 10th percentile score of each subscale was used to identify children at a potential risk of developmental problem/delay, i.e., if scores on any subscale were less than or equal to the 10th percentile, the child was classified as having a developmental problem/delay. Additional file 1: Table S1 presents the rates of potential developmental problem assessed at 4 years of age in the study.

\section{Covariates and potential confounders}

Trained interviewers used a structured questionnaire at enrollment to collect information on maternal age, education, height, pre-pregnancy weight, parity, health status, per capita household income, and lifestyle. Body mass index (BMI) was calculated as body weight in kilograms divided by squared body height in meters. The information on child's sex and gestational age was extracted from the study hospital's medical records. Potential confounders were identified based on previous literature of potential determinants of early childhood development, available data in the present study, and results of bivariate analyses examining the relationship with neuropsychological problems $(P<0.20)$. Maternal age at enrollment (years), pre-pregnancy BMI $\left(\mathrm{kg} / \mathrm{m}^{2}\right)$, parity, per capita household income $(<4000,4000-8000$, and $>8000 \mathrm{CNY} /$ month), passive smoker (yes/no), gestational age (weeks), and child's sex (Boy/Girl) were included as covariates in the final model.

\section{Statistical analysis}

We first described and compared the demographic characteristics of included and excluded mother-infant pairs. The distributions of prenatal plasma concentrations of PFASs were presented by geometric means (GMs), SD, and percentiles. Risk ratios (RRs) and associated 95\% confidence intervals (CIs) were estimated for the association between each PFAS and each developmental subscale of ASQ using Poisson regression analysis with robust variance estimates [20]. Prenatal PFAS concentrations were natural $\log (\ln )$ transformed to approximate a normal distribution for regression analysis and were treated as continuous independent variables in all models. Values below the LOD were replaced with $\mathrm{LOD} / \sqrt{ } 2$.

Considering that a previous study reported the sexspecific effect of PFASs on neurobehavioral problems [21], we introduced a cross product term for child's sex with each individual PFAS to evaluate potential interaction effects. As several interaction items showed statistical significance $(P<0.10)$, we performed stratified analyses by child's sex. PFAS concentrations were also categorized into tertiles according to its distribution in the subjects and were analyzed using Poisson regression. The lowest tertile was used as the reference group.

Additionally, we fit generalized additive models to investigate a potential nonlinear relationships between maternal PFAS concentration and neurobehavioral problems, and visually inspected plots of the smoothed data. Several associations between ln-transformed PFASs and children's neuropsychological development showed nonlinear (Additional file 1: Figure S1-S5). In order to make our results comparable to other studies and considering that the aim of our study was inference rather than 
prediction, we still primarily presented the results of linear models (PFAS concentrations as continuous and categorical variables, respectively),

The Statistical Analysis System (SAS, version 9.3; SAS Institute, Inc., Cary, NC, USA) was used for statistical analysis. $P$ values $<0.05$ from two-tailed tests were considered statistically significant.

\section{Results}

Table 1 presents characteristics of mother-child pairs included and excluded in the study. The mean age of the included mothers at recruitment was 27.9 years, and 18.7 and $5.9 \%$ of them were underweight and overweight, respectively. Included parents tended to have high educational attainment, with $78.0 \%$ having college or above degrees. Among included mothers, the majority were nulliparous and had household income per capita $>4000 \mathrm{CNY} /$ month, and over $40 \%$ of them were exposed to secondhand smoke during pregnancy, while only $0.87 \%$ of them reported alcohol consumption during pregnancy. About $18.6 \%$ of included mothers had depressive symptoms during pregnancy. The mean gestational weeks of included mothers at birth of children was 39.5 weeks and $55.7 \%$ of children were boys. The characteristics of excluded mother-pairs were comparable with those included, except that the proportion of nulliparous women was slightly higher among the included than those excluded (86.9\% vs. $82.7 \%$ ).

PFHxS, PFOS, PFOA, PFNA, PFDA, PFUdA, and PFDoA were detected in all maternal plasma samples, while PFTrDA was detected in $90.6 \%$ of samples. PFOA had the highest exposure levels $(\mathrm{GM}=20.0 \mathrm{ng} / \mathrm{ml})$, followed by PFOS $(\mathrm{GM}=10.8 \mathrm{ng} / \mathrm{ml})$. The concentrations of PFHxS, PFDA, PFNA, and PFUdA were one order of magnitude lower than those of PFOA and PFOS, while those of PFDoA and PFTrDA were two orders of magnitude lower (Table 2). Prenatal PFAS concentrations were similarly distributed in girls and boys (Additional file 1: Table S2).

Table 3 and Fig. 1 present associations between maternal ln-transformed PFAS concentrations (as continuous variables) and children's neurodevelopmental problems at 4 years of age. There was a pattern of higher risks of developmental problem in personal-social skills associated with higher prenatal plasma concentrations of PFHxS, PFOS, PFOA, PFNA, PFDA, and PDUdA, with significant associations for PFNA and PFDA (per natural $\log$ unit increase: $R_{\mathrm{PFNA}}=1.92,95 \% \mathrm{CI}: 1.21,3.05$; RR PFDA $=1.66,95 \%$ CI: 1.17, 2.37). Children with higher prenatal PFTrDA concentrations tended to have a slightly higher risk of developmental problem in communication, with borderline significance $(R R=1.16$ for per natural log unit increase, 95\% CI: 0.99, 1.36), but the association was not observed for other PFASs. No statistically significant association was observed between maternal PFAS concentrations and developmental problems in gross motor, fine motor, and problem solving skills.

When evaluating the potential effect modifications by child's sex, some interaction items of maternal PFAS concentrations and child's sex were statistically significant $(p<0.10)$ in the models examining PFOA and gross motor, PFNA and fine motor, and PFOS/PFOA and personal-social skills (Additional file 1: Table S3). The consistent pattern of higher risk of developmental problems in personal-social skills associated with most PFASs shown in Table 3 was mainly observed among girls (Table 4). Among these associations, those for PFOS, PFOA, PFNA, PFDA, PFUdA, and PFDoA concentrations were statistically significant (per natural log unit increase of PFAS concentrations: $\mathrm{RR}_{\mathrm{PFOS}}=2.56,95 \% \mathrm{CI}$ : $1.20,5.45 ; \mathrm{RR}_{\mathrm{PFOA}}=9.00,95 \%$ CI: 3.82, 21.21; $\mathrm{RR}_{\mathrm{PFNA}}=$ 3.11, 95\% CI: 1.36, 7.13; $\mathrm{RR}_{\text {PFDA }}=2.20$, 95\% CI: 1.21 , 4.00; $\mathrm{RR}_{\mathrm{PFUdA}}=2.44,95 \% \mathrm{CI}: 1.14,5.20 ; \mathrm{R}_{\text {PFDoA }}=1.62$, 95\% CI: 1.04, 2.54). There were no significant associations between PFAS concentrations and problems in other subscales in girls (Table 4). No clear association between PFAS concentrations and problems in each subscale was observed in boys (Table 4). Only boys with higher prenatal PFOA concentrations had a decreased risk of developmental problems in gross motor skills (per natural $\log$ unit increase: $\mathrm{RR}=0.47,95 \% \mathrm{CI}$ : 0.25 , 0.89) (Table 4).

We further examined the associations between maternal PFAS concentration as categorical variables and developmental problems by child's sex (Table 5). Generally, the models using categorized PFAS variables showed similar results as reported in the main analyses. In girls, there was a consistent pattern of increased risk of problems in personal-social skills associated with higher maternal PFAS concentrations except PFTrDA, although the estimates became imprecise. For PFOA, the regression model did not converge because there was no child with developmental problem in the lowest tertile group. A linear trend was observed between tertiles of PFOS, PFNA, PFDA, and PFDoA and problems in the subscale $(\mathrm{P}$ for trend $=0.0027,0.0417,0.0110$, and 0.0159, respectively, Table 5). In addition, prenatal PFNA concentrations were associated with a decreased risk of communication problems; adjusted RRs were 0.73 (95\%CI: $0.41,1.32)$ for the middle tertile and $0.50(95 \%$ CI: $0.26,0.94)$ for the highest tertile ( $\mathrm{P}$ for trend = 0.0292). There were no clear associations between maternal PFAS concentrations and problems in each subscale among boys. However, boys with higher maternal PFTrDA concentrations were more likely to have communication problem; the adjusted RRs was 1.53 (95\%CI: $0.92,2.55)$ for middle tertile and 1.83 (95\%CI: 1.08, 3.12) 
Table 1 Characteristics of included and excluded mother-child pairs

\begin{tabular}{|c|c|c|c|}
\hline Characteristics & $\begin{array}{l}\text { Included }(N=533) \\
\mathrm{n}(\%) / \text { Mean } \pm \text { SD }\end{array}$ & $\begin{array}{l}\text { Excluded }(N=692) \\
n(\%) / \text { Mean } \pm S D\end{array}$ & $\begin{array}{l}P \text {-value of Student's t-test } \\
\text { or Chi-square test }\end{array}$ \\
\hline \multicolumn{4}{|c|}{ Maternal age at enrollment (years) } \\
\hline Mean \pm SD & $27.9 \pm 3.4$ & $27.8 \pm 3.4$ & 0.7451 \\
\hline$<25$ & $81(15.2)$ & $103(14.9)$ & 0.7900 \\
\hline $25-30$ & $299(56.1)$ & $404(58.4)$ & \\
\hline$\geq 30$ & $153(28.7)$ & $185(26.7)$ & \\
\hline \multicolumn{4}{|c|}{ Maternal pre-pregnancy BMI $\left(\mathrm{kg} / \mathrm{m}^{2}\right)$} \\
\hline Mean \pm SD & $20.6 \pm 2.4$ & $20.4 \pm 2.4$ & 0.1905 \\
\hline$<18.5$ & $98(18.7)$ & $146(21.5)$ & 0.2112 \\
\hline $18.5-24.9$ & $395(75.4)$ & $506(74.4)$ & \\
\hline$\geq 25$ & $31(5.9)$ & $28(4.1)$ & \\
\hline \multicolumn{4}{|l|}{ Maternal education } \\
\hline Blow high school & $41(7.7)$ & $78(11.3)$ & 0.1064 \\
\hline High School & $76(14.3)$ & $99(14.3)$ & \\
\hline College or above & $415(78.0)$ & $514(74.4)$ & \\
\hline \multicolumn{4}{|l|}{ Paternal education } \\
\hline Blow high school & $28(5.25)$ & $57(8.28)$ & 0.9430 \\
\hline High School & $76(14.26)$ & $119(17.3)$ & \\
\hline College or above & $429(80.49)$ & $512(74.42)$ & \\
\hline \multicolumn{4}{|c|}{ Per capita household income (CNY) } \\
\hline$<4000$ & $113(21.3)$ & $140(20.6)$ & 0.0282 \\
\hline $4000-8000$ & $212(40.0)$ & $277(40.8)$ & \\
\hline$>8000$ & $205(38.7)$ & $262(38.6)$ & \\
\hline \multicolumn{4}{|l|}{ Parity } \\
\hline Nulliparous & $459(86.9)$ & $569(82.7)$ & 0.0432 \\
\hline Multiparous & $69(13.1)$ & $119(17.3)$ & \\
\hline \multicolumn{4}{|c|}{ Maternal alcohol consumption during pregnancy } \\
\hline No & $454(99.13)$ & $508(98.64)$ & 0.4743 \\
\hline Yes & $4(0.87)$ & $7(1.36)$ & \\
\hline \multicolumn{4}{|c|}{ Maternal passive smoking before conception } \\
\hline No & $307(57.8)$ & $420(60.9)$ & 0.2811 \\
\hline Yes & $224(42.2)$ & $270(39.1)$ & \\
\hline \multicolumn{4}{|c|}{ Maternal prenatal depressive symptoms } \\
\hline No & $434(81.43)$ & $551(79.62)$ & 0.4309 \\
\hline Yes & $99(18.57)$ & $141(20.38)$ & \\
\hline \multicolumn{4}{|l|}{ Sex of child } \\
\hline Boy & $297(55.7)$ & $370(53.7)$ & 0.4816 \\
\hline Girl & $236(44.3)$ & $319(46.3)$ & \\
\hline \multicolumn{4}{|c|}{ Gestational age (weeks) } \\
\hline Mean \pm SD & $39.5 \pm 1.3$ & $39.51 \pm 1.5$ & 0.6182 \\
\hline$<37$ & $19(3.6)$ & $26(3.8)$ & 0.8640 \\
\hline$\geq 37$ & $513(96.4)$ & $666(96.2)$ & \\
\hline
\end{tabular}

Missing data: Included: pre-pregnancy BMI ( $n=9)$, maternal education $(n=1)$, per capita household income $(n=3)$, parity $(n=5)$, maternal alcohol consumption during pregnancy $(n=75)$ and maternal passive smoking before conception $(n=2)$; Excluded: pre-pregnancy BMI $(n=12)$, maternal education $(n=1)$, paternal education $(n=4)$, per capita household income $(n=13)$, parity $(n=4)$, maternal alcohol consumption during pregnancy $(n=177)$, maternal passive smoking before conception $(n=2)$, and maternal prenatal depressive symptoms $(n=1)$ 
Table 2 Maternal PFASs concentrations ( $\mathrm{ng} / \mathrm{mL}$ ) at $12-16$ gestational weeks $(N=533)$ in Shanghai, China

\begin{tabular}{|c|c|c|c|c|c|c|c|c|}
\hline \multirow[t]{2}{*}{ PFAS } & \multirow[t]{2}{*}{ LOD } & \multirow{2}{*}{$\begin{array}{l}>\text { LOD (N } \\
\%)\end{array}$} & \multirow{2}{*}{$\begin{array}{l}\text { GM } \\
\text { (GSD) }\end{array}$} & \multicolumn{5}{|c|}{ Percentiles } \\
\hline & & & & 5th & 25th & 50th & 75th & 95th \\
\hline PFHXS & 0.015 & $533(100)$ & $2.7(1.6)$ & 1.4 & 2.1 & 2.8 & 3.5 & 5.7 \\
\hline PFOS & 0.02 & $533(100)$ & $10.8(1.8)$ & 4.5 & 7.6 & 10.8 & 15.8 & 25.2 \\
\hline PFOA & 0.01 & $533(100)$ & $20.0(1.6)$ & 9.3 & 15.3 & 19.9 & 27.4 & 38.9 \\
\hline PFNA & 0.02 & $533(100)$ & $1.8(1.6)$ & 0.8 & 1.3 & 1.8 & 2.5 & 3.9 \\
\hline PFDA & 0.01 & $533(100)$ & $2.1(1.9)$ & 0.7 & 1.4 & 2.1 & 3.2 & 6.3 \\
\hline PFUdA & 0.01 & $533(100)$ & $1.6(1.9)$ & 0.5 & 1.0 & 1.6 & 2.5 & 4.4 \\
\hline PFDoA & 0.015 & $533(100)$ & $0.1(2.9)$ & LOD & 0.1 & 0.1 & 0.2 & 0.4 \\
\hline PFTrDA & 0.02 & 483 (90.6) & $0.1(2.9)$ & LOD & 0.1 & 0.1 & 0.2 & 0.4 \\
\hline
\end{tabular}

LOD Limit of detection, GM Geometric mean, GSD Geometric

standard deviation

for the highest tertile ( $\mathrm{P}$ for trend $=0.0218$ ). Boys in the middle tertile of maternal PFUdA concentrations had more problems in fine motor scale $(\mathrm{RR}=2.19,95 \% \mathrm{CI}$ : 1.16, 4.17) (Table 5).

\section{Discussion}

We found that girls with higher maternal concentrations of PFHxS, PFOS, PFOA, PFNA, PFDA, PFUdA, and PFDoA tended to have more problems in personal-social skills, while there was little evidence for consistent associations in boys.

In the study, median concentrations for maternal PFOS and PFOA were 10.8 and $19.9 \mathrm{ng} / \mathrm{ml}$, respectively, which are one of the highest levels reported among pregnant women during the similar period (around 2012) compared to those of the studies conducted in the US (2.4 and $1.1 \mathrm{ng} / \mathrm{ml})$ [16], Canada ( 4.6 and $1.7 \mathrm{ng} / \mathrm{ml})$ [22], Denmark (8.23 and $2.0 \mathrm{ng} / \mathrm{ml}$ ) [23], Australia (1.99 ad $0.86 \mathrm{ng} / \mathrm{ml}$ ) [24], and Japan (3.52 and $1.27 \mathrm{ng} / \mathrm{ml}$ ) [25], especially for PFOA. However, compared to the studies on prenatal PFAS concentrations and child's neurodevelopment where the samples were collected mainly around 2000, PFOS concentrations were lower than most of the studies (ranged from 13.2 to $34.4 \mathrm{ng} / \mathrm{ml}$ ) [10, 26] except the Hokkaido study in Japan [12, 27], while PFOA concentrations were much higher (ranged from 1.2 to $5.6 \mathrm{ng} / \mathrm{ml}$ in previous studies) [12, 28]. Different from other studies where PFOS has the highest concentrations, PFOA was the most predominant compound in the present study, which was also observed in another cohort study in Shanghai [29]. This may be explained by the findings that PFOA is the most prevalent compound in the surface water of Shanghai, accounting for $51-86 \%$ of total PFAS concentrations [30].

Results from epidemiological studies on neurodevelopmental impact of in utero PFAS exposure are inconsistent $[10,28,31,32]$. A cohort study from Taiwan showed that prenatal exposure to PFOS might affect child neurodevelopment, especially gross motor development at 2 years of age [32]. Among 432 mother-daughter pairs from the Avon Longitudinal cohort, a $1 \mathrm{ng} / \mathrm{mL}$ increase in PFOS was associated with a 3.82-point (95\% CI: $6.18,-1.47)$ lower vocabulary score at 15 months and a 0.80 -point (95\% CI: $-1.74,0.14)$ lower language score at 38 months in daughters of mothers aged $<25$ years [33]. A birth cohort study between 2002 and 2005 suggested an association between prenatal PFOA exposure and neurodevelopmental delay in 6-month-old females, as measured by mental scales of the second edition of the Bayley Scales of Infant Development [12]. In Oulhote et al.'s study, cross-sectional analyses at 7 years of age showed possible sex-dimorphic associations between PFAS concentrations and the Strengths and Difficulties Questionnaire (SDQ) scores; girls had consistently positive associations with SDQ scores, whereas boys exhibited a pattern of negative or null associations [9]. However, in a prospective study from the DNBC, maternal plasma levels of PFOA or PFOS were neither associated with mental developmental nor fine and gross motor developmental milestones in infants [28], and there was no association between PFAS concentration and behavioral and motor coordination problems at 7 years of age [10]. In a Norwegian birth cohort study, PFOA or PFOS measured in breast milk was not associated with child neuropsychological development assessed by ASQ at 12 and 24 months [31]. Moreover, behavioral development assessed by the Infant-Toddler Symptom Checklist (ITSC) found no consistent increase in behavioral problems at 12 and 24 months [31]. The inconsistent findings between the current study and previous studies may be due to differences in screening tools, children's ages at assessment, and PFAS compounds measured and their concentrations [10, 28, 31].

The mechanism of the effect of PFASs on neurobehavioral development remains unclear. In animal studies, some PFASs may affect the cholinergic or dopaminergic system, resulting in altered responses to nicotine or imbalanced expression of the acetylcholine/dopamine phenotype [6]. PFASs also affect synaptogenesis and functional protein levels during neuron growth [34]. PFOA and PFOS significantly increased the levels of synaptophysin and tau in the cerebral cortex and hippocampus. Because these proteins are important for normal brain development, altered levels during a critical period of brain growth spurts could be one of the mechanisms of behavioral defects [34]. Other possible mechanisms include the endocrine-disrupting properties of PFASs in glucocorticoid, sex hormone [27] and thyroid hormone balance $[35,36]$. Prenatal and postnatal exposure to PFASs interferes with thyroid hormone balance in humans, resulting in higher thyroid-stimulating hormone, decreased total/free triiodothyronine, and decreased total/ 


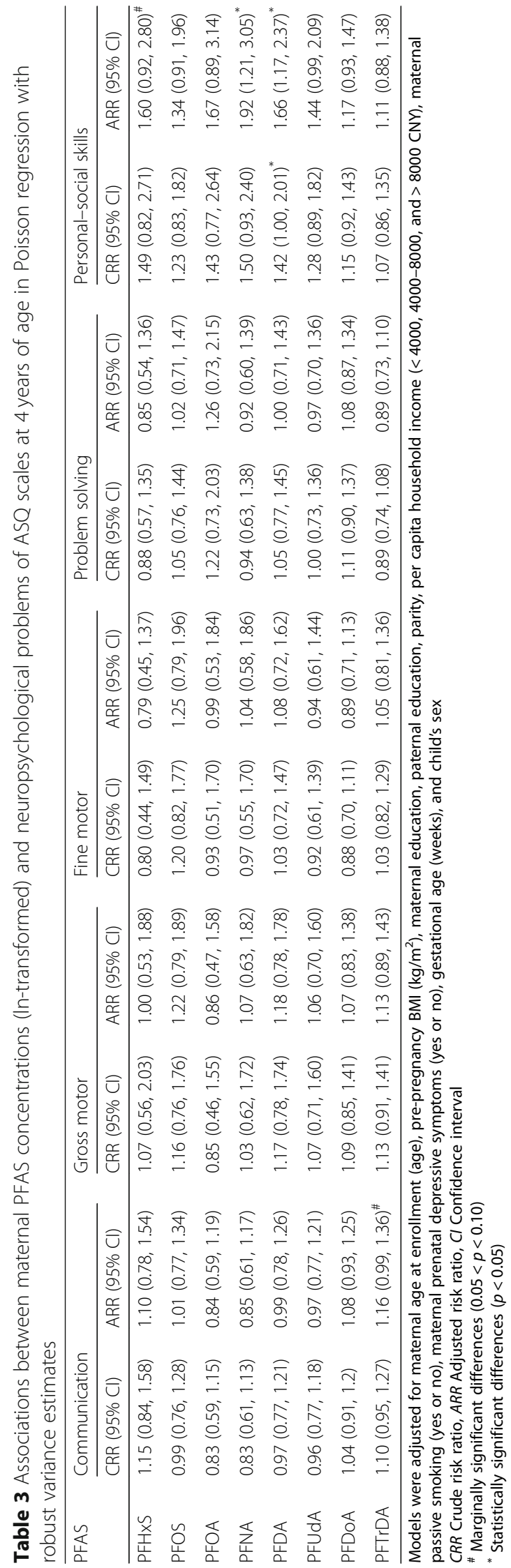



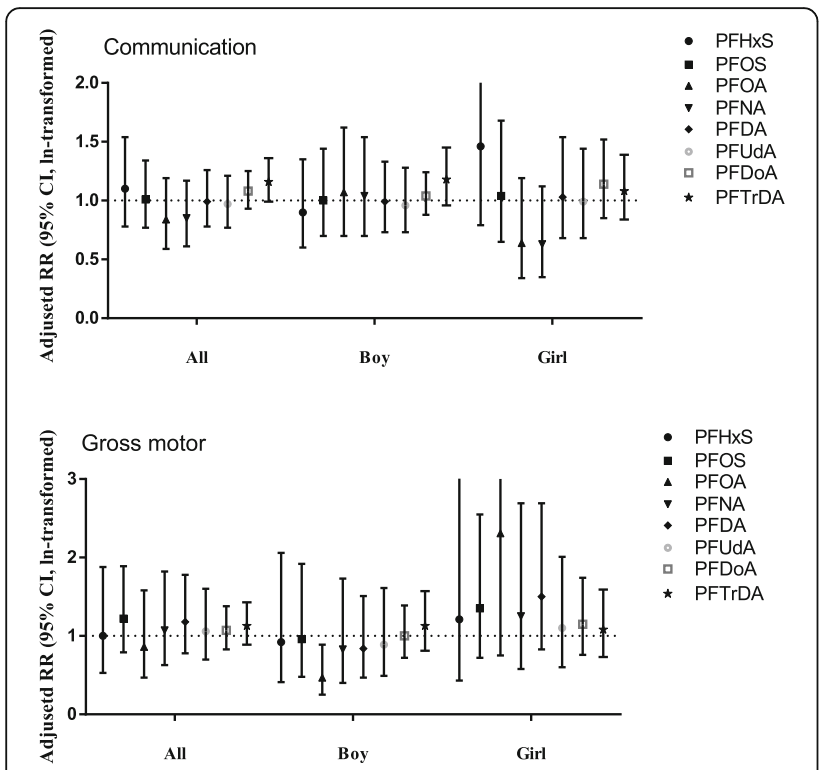

- PFHxS

- PFOS

- PFOA

- PFNA

PFDA

PFUdA

* PFTrDA

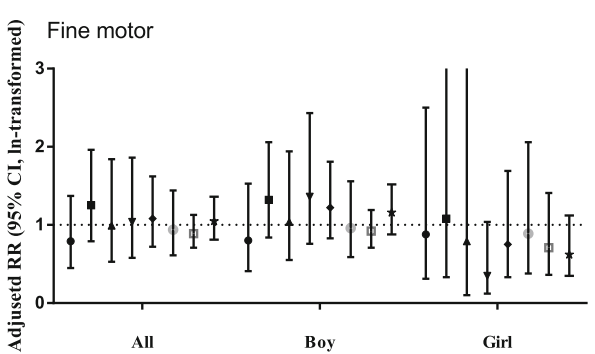

All

Boy

Girl

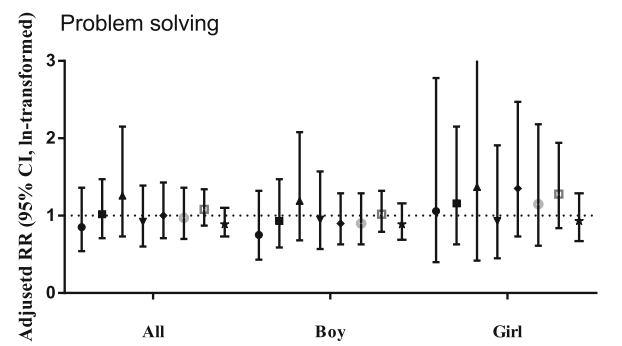

- PFHXS

- PFOS

PFOA

PFNA

- PFUdA

- PFDoA

* PFTrDA

Boy

Gir

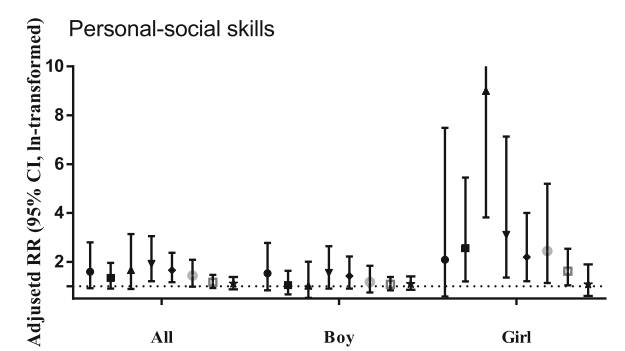

Fig. 1 Associations between maternal PFAS concentrations (Intransformed) and neuropsychological problems of ASQ scales at 4 years of age in Poisson regression with robust variance estimates

free thyroxine levels [37-39], which may play a role in how PFASs affect human neurodevelopment.

Additionally, we found a consistent pattern of adverse effects on personal-social skills in girls but not in boys following prenatal PFAS exposure. Although chance findings may be incompletely excluded, mechanism investigations are warranted to understand the sex-specific association. In previous studies, prenatal PFOA exposure was associated with decreased Mental Developmental indices scores of female infants at 6 months of age [12], and PFOS exposure was associated with poorer metacognition scores only among school-aged girls [26]. These findings are in line with our results on personalsocial skills which evaluate children's abilities of selfhelping and interacting with others. Human studies suggested that prenatal PFOA/PFOS exposure was significantly associated with testosterone/estradiol in male infants, progesterone levels, glucocorticoid levels, and DHEA levels in cord blood samples of both sexes [27, 40]. In addition, effects of PFAS exposure on thyroid hormone homeostasis may differ across sexes [41]. The hormonal effects of PFAS may differently affect the neurobehavioral development of males or females. However, whether the sex-specific difference is inherited due to sex or due to hormonal effects of PFAS exposure warrants further investigation.

One strength of our study is that the prospective design provides strong causality between PFAS exposure levels and child neurodevelopment. We measured two of the most frequently detected PFASs, PFOS and PFOA, as well as other PFAS compounds to provide a profile of the effects of commonly detected PFAS compounds. However, some potential limitations of the current study should be mentioned. First, there was considerable loss to follow-up for neurodevelopmental assessment during the study period, which increased the potential for selection bias. However, the characteristics of subjects in the original cohort were similar to those in the final sample in terms of maternal age, pre-pregnancy BMI, parity, and gestational age. Thus, the loss to follow-up was less likely to lead to substantial bias. Second, parental intelligence quotient (IQ) may affect the children's ASQ score, however, the information about parental IQ has not been collected in the study. We adjusted for parental education in the models, which may partially control for the confounding effects of parental IQ. In addition, the relationship between PFASs and ASQ measures may have been confounded by postnatal environmental risk factors $[42,43]$. The confounding effect of uncollected factors, e.g., child sleepiness and maternal selfregulation, cannot be adjusted for. Third, multiple comparisons may also be of concern because we examined the associations between eight PFASs and five subscales. However, for our main findings on the associations 


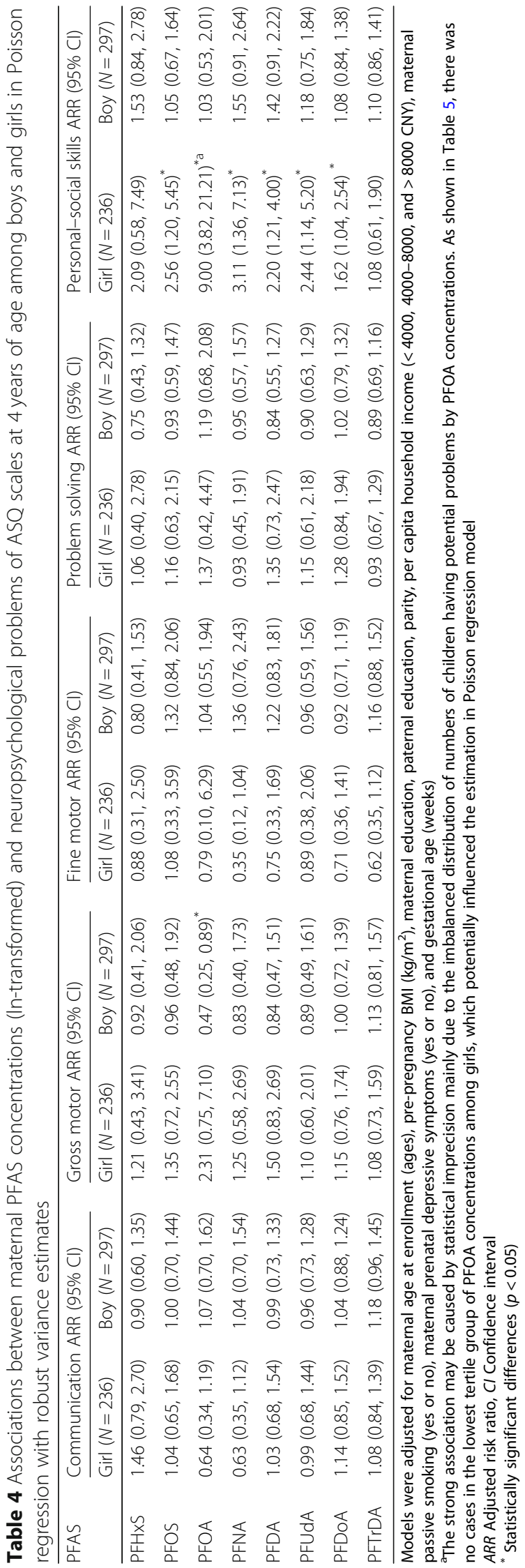




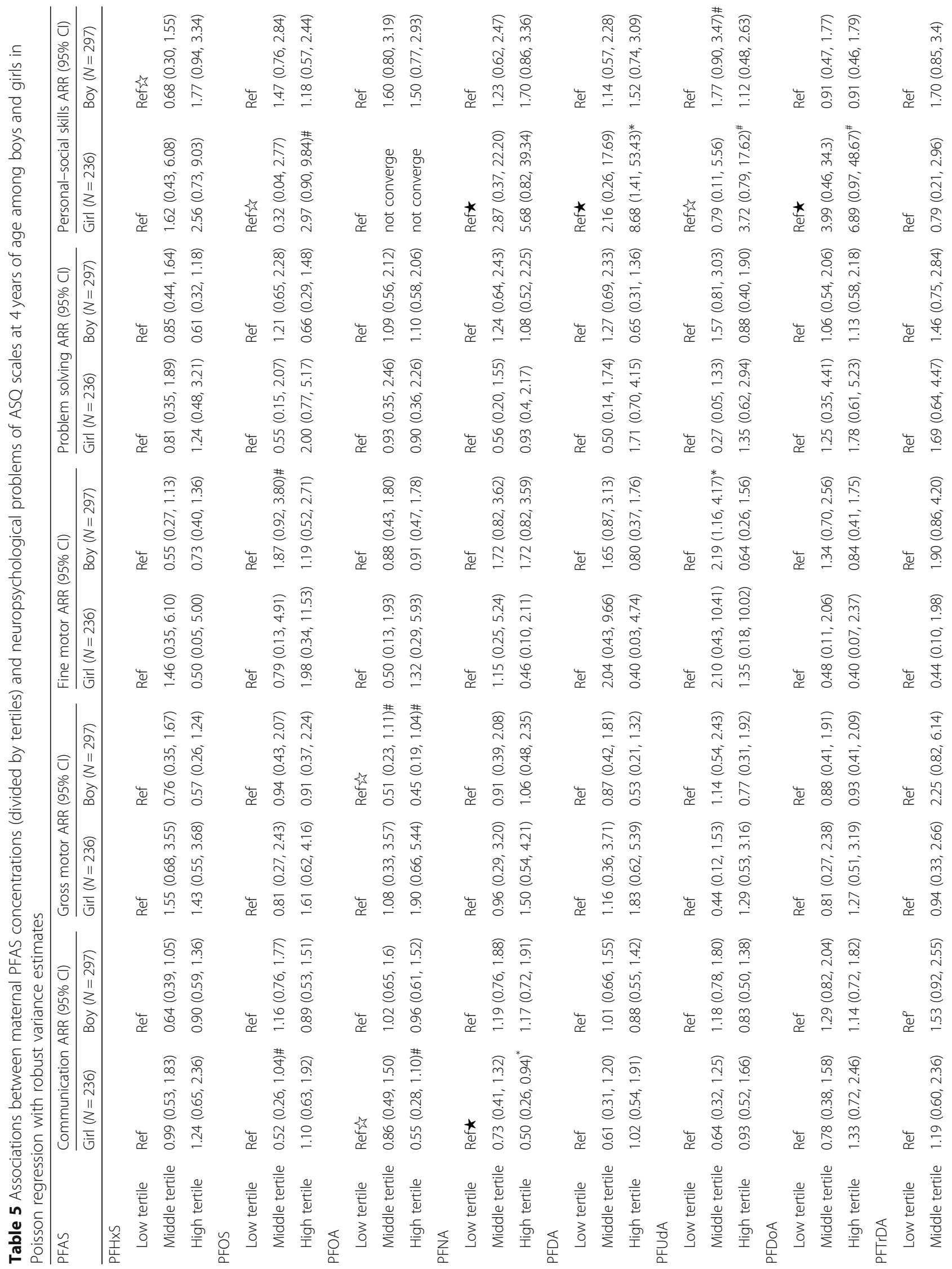




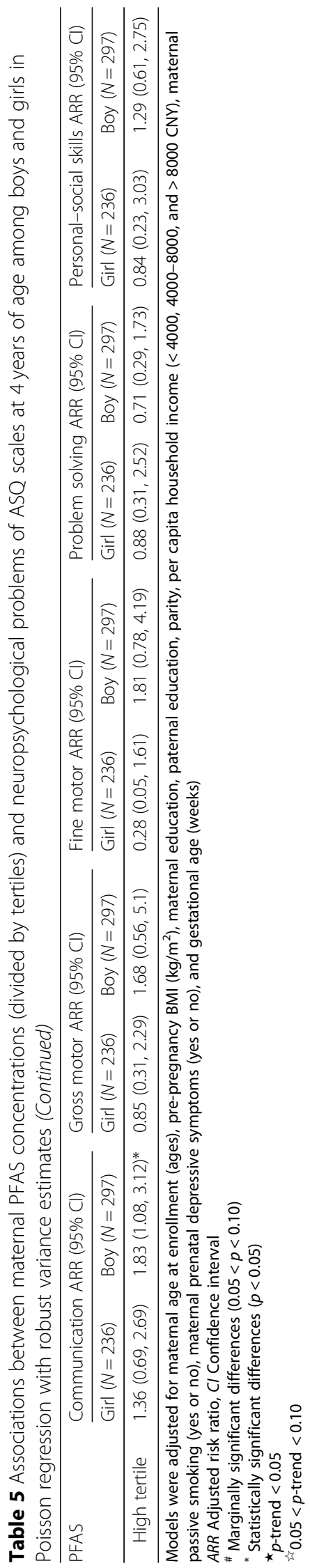


between PFAS concentrations and personal-social skills among girls, patterns were consistent across PFAS compounds, which were less likely to be due to chance alone.

\section{Conclusions}

Maternal PFAS concentrations during pregnancy were inversely associated with neuropsychological development assessed by ASQ in 4-year-old children. Further investigation of the underlying mechanism of the effect of prenatal PFAS exposure on neuropsychological development is needed.

\section{Additional file}

Additional file 1: Table S1. The rates of potential developmental problem assessed at 4 years of age by the Age and Stage Questionnaire (ASQ). Table S2. Maternal PFASs concentrations $(\mathrm{ng} / \mathrm{mL})$ at 12-16 gestational weeks stratified by child sex. Table S3. $P$-values of interaction item (PFASs*child sex) in the associations between maternal PFASs concentrations and child's neurobehavioral problems assessed by ASQ at 4 years of age*. Figure S1. Adjusted generalized additive model plots of In-transformed PFAS concentrations with developmental problems in communication among offspring. Figure S2. Adjusted generalized additive model plots of In-transformed PFAS concentrations with offspring developmental problems in Gross motor among offspring. Figure S3. Adjusted generalized additive model plots of In-transformed PFAS concentrations with developmental problems in Fine motor among offspring. Figure S4. Adjusted generalized additive model plots of Intransformed PFAS concentrations with developmental problems in Problem solving among offspring. Figure S5. Adjusted generalized additive model plots of In-transformed PFAS concentrations with developmental problems in Personal-social skills among offspring. (DOCX 15944 kb)

\section{Acknowledgements}

This work was supported by grants from the National Key Research and Development Program of China (2018YFC1002801, 2016YFC1000505, 2017YFC1600500); the Science and Technology Commission of Shanghai Municipality (16ZR1430100); the National Natural Science Foundation of China (21628701); Shanghai municipal health commission (201840184); Health Commission of Zhejiang Province (2018KY812), Health Commission of Jiaxing City (2016BY58088, 2016A05), and Innovation-oriented Science and Technology Grant from NHC Key Laboratory of Reproduction Regulation (CX2017-06)

\section{Authors' contributions}

WY, HL and MM conceived and designed the study. JN, YT and HL performed data analysis and drafted the article. WY, MM, YR, HX, XSu, HH, XSo, LY and SW revised the manuscript and critically discussed the results. All authors were involved in interpreting the data and approved the final article.

\section{Funding}

None.

\section{Availability of data and materials}

The datasets used in the current study are available from the corresponding authors on reasonable request.

\section{Ethics approval and consent to participate}

The study was approved by the ethical review committee of Shanghai Institute of Planned Parenthood Research (SIPPR). The study was conducted according to the Declaration of Helsinki. All mothers gave their written informed consent for themselves and their children before enrollment and at each follow-up.
Consent for publication

Not applicable.

\section{Competing interests}

The authors declare that they have no competing interests.

\section{Author details}

${ }^{1}$ The First People's Hospital of Jianshan, Jiaxing, Zhejiang Province, China. ${ }^{2} \mathrm{NHC}$ Key Lab of Reproduction Regulation (Shanghai Institute of Planned Parenthood Research), Fudan University, Shanghai, China. ${ }^{3}$ Department of Pharmaceutical Outcomes \& Policy, College of Pharmacy, University of Florida, 1225 Center Drive, HPNP 3338, Gainesville, FL 32610, USA. ${ }^{4}$ Department of Epidemiology, College of Public Health and Health Professions and College of Medicine, University of Florida, 2004 Mowry Road, Gainesville, FL 32610, USA. ${ }^{5}$ National Reference Laboratory of Dioxin, Institute of Health Inspection and Detection, Hubei Provincial Academy of Preventive Medicine, Hubei Provincial Center for Disease Control and Prevention, Wuhan 430079, China. ${ }^{6}$ Department of Public Educaion, Weifang Medical University, 7166 Baotong west Road, Weifang 261053, Shandong Province, China. ${ }^{7}$ Department of Health Statistics, School of Public Health and Management, Weifang Medical University, 7166 Baotong west Road, Weifang 261053, Shandong Province, China.

Received: 5 January 2019 Accepted: 31 May 2019

Published online: 13 June 2019

References

1. Post GB, Cohn PD, Cooper KR. Perfluorooctanoic acid (PFOA), an emerging drinking water contaminant: a critical review of recent literature. Environ Res. 2012;116:93-117.

2. Olsen GW, Burris JM, Ehresman DJ, Froehlich JW, Seacat AM, Butenhoff JL, Zobel LR. Half-life of serum elimination of perfluorooctanesulfonate, perfluorohexanesulfonate, and perfluorooctanoate in retired fluorochemical production workers. Environ Health Perspect. 2007;115(9):1298-305.

3. Houde M, Martin JW, Letcher RJ, Solomon KR, Muir DC. Biological monitoring of polyfluoroalkyl substances: a review. Environ Sci Technol. 2006:40(11):3463-73.

4. Lau C, Anitole K, Hodes C, Lai D, Pfahles-Hutchens A, Seed J. Perfluoroalkyl acids: a review of monitoring and toxicological findings. Toxicol Sci. 2007: 99(2):366-94.

5. Apelberg BJ, Witter FR, Herbstman JB, Calafat AM, Halden RU, Needham LL, Goldman LR. Cord serum concentrations of perfluorooctane sulfonate (PFOS) and perfluorooctanoate (PFOA) in relation to weight and size at birth. Environ Health Perspect. 2007:115(11):1670-6.

6. Johansson N, Fredriksson A, Eriksson P. Neonatal exposure to perfluorooctane sulfonate (PFOS) and perfluorooctanoic acid (PFOA) causes neurobehavioural defects in adult mice. Neurotoxicology. 2008;29(1):160-9.

7. Butenhoff JL, Ehresman DJ, Chang SC, Parker GA, Stump DG. Gestational and lactational exposure to potassium perfluorooctanesulfonate (K+PFOS) in rats: developmental neurotoxicity. Reprod Toxicol (Elmsford, NY). 2009;27(34):319-30.

8. Stein CR, Savitz DA, Bellinger DC. Perfluorooctanoate and neuropsychological outcomes in children. Epidemiology (Cambridge, Mass). 2013:24(4):590-9.

9. Oulhote Y, Steuerwald U, Debes F, Weihe P, Grandjean P. Behavioral difficulties in 7-year old children in relation to developmental exposure to perfluorinated alkyl substances. Environ Int. 2016;97:237-45.

10. Fei C, Olsen J. Prenatal exposure to perfluorinated chemicals and behavioral or coordination problems at age 7 years. Environ Health Perspect. 2011; 119(4):573-8.

11. Liew Z, Ritz B, von Ehrenstein OS, Bech BH, Nohr EA, Fei C, Bossi R, Henriksen TB, Bonefeld-Jorgensen EC, Olsen J. Attention deficit/hyperactivity disorder and childhood autism in association with prenatal exposure to perfluoroalkyl substances: a nested case-control study in the Danish National Birth Cohort. Environ Health Perspect. 2015;123(4):367-73.

12. Goudarzi H, Nakajima S, Ikeno T, Sasaki S, Kobayashi S, Miyashita C, Ito S, Araki A, Nakazawa H, Kishi R. Prenatal exposure to perfluorinated chemicals and neurodevelopment in early infancy: the Hokkaido study. Sci Total Environ. 2016;541:1002-10

13. Hoyer BB, Ramlau-Hansen CH, Obel C, Pedersen HS, Hernik A, Ogniev V, Jonsson BA, Lindh CH, Rylander L, Rignell-Hydbom A, et al. Pregnancy 
serum concentrations of perfluorinated alkyl substances and offspring behaviour and motor development at age 5-9 years--a prospective study. Environ Health. 2015;14:2.

14. Tian Y, Zhou Y, Miao M, Wang Z, Yuan W, Liu X, Wang X, Wang Z, Wen S, Liang $H$. Determinants of plasma concentrations of perfluoroalkyl and polyfluoroalkyl substances in pregnant women from a birth cohort in Shanghai, China. Environ Int. 2018;119:165-73.

15. Cariou R, Veyrand B, Yamada A, Berrebi A, Zalko D, Durand S, Pollono C, Marchand P, Leblanc JC, Antignac JP, et al. Perfluoroalkyl acid (PFAA) levels and profiles in breast milk, maternal and cord serum of French women and their newborns. Environ Int. 2015;84:71-81.

16. Starling AP, Adgate JL, Hamman RF, Kechris K, Calafat AM, Ye X, Dabelea D Perfluoroalkyl substances during pregnancy and offspring weight and adiposity at birth: examining mediation by maternal fasting glucose in the healthy start study. Environ Health Perspect. 2017;125(6):067016.

17. Xia R, Jin L, Li D, Liang H, Yang F, Chen J, Yuan W, Miao M. Association between paternal alcohol consumption before conception and Anogenital distance of offspring. Alcohol Clin Exp Res. 2018;42(4):735-42.

18. Squires JBD. Ages \& Stages Questionnaires[R], Third Edition (ASQ-3[TM]): A Parent-Completed Child-Monitoring System. Baltimore: Brookes Publishing Company; 2009.

19. Wei M, Bian X, Squires J, Yao G, Wang X, Xie H, Song W, Lu J, Zhu C, Yue H, et al. Studies of the norm and psychometrical properties of the ages and stages questionnaires, third edition, with a Chinese national sample. Zhonghua Er Ke Za Zhi. 2015;53(12):913-8.

20. Zou G. A modified poisson regression approach to prospective studies with binary data. Am J Epidemiol. 2004;159(7):702-6.

21. Stein CR, Savitz DA, Bellinger DC. Perfluorooctanoate exposure in a highly exposed community and parent and teacher reports of behaviour in 6-12year-old children. Paediatr Perinat Epidemiol. 2014;28(2):146-56.

22. Fisher M, Arbuckle TE, Liang CL, LeBlanc A, Gaudreau E, Foster WG, Haines D, Davis K, Fraser WD. Concentrations of persistent organic pollutants in maternal and cord blood from the maternal-infant research on environmental chemicals (MIREC) cohort study. Environ Health. 2016;15(1):59.

23. Bjerregaard-Olesen C, Bach CC, Long M, Ghisari M, Bech BH, Nohr EA, Henriksen TB, Olsen J, Bonefeld-Jorgensen EC. Determinants of serum levels of perfluorinated alkyl acids in Danish pregnant women. Int J Hyg Environ Health. 2016:219(8):867-75.

24. Callan AC, Rotander A, Thompson K, Heyworth J, Mueller JF, Odland JO, Hinwood AL. Maternal exposure to perfluoroalkyl acids measured in whole blood and birth outcomes in offspring. Sci Total Environ. 2016;569-570: 1107-13.

25. Okada E, Kashino I, Matsuura H, Sasaki S, Miyashita C, Yamamoto J, Ikeno T, Ito YM, Matsumura T, Tamakoshi A, et al. Temporal trends of perfluoroalkyl acids in plasma samples of pregnant women in Hokkaido, Japan, 2003-2011. Environ Int. 2013;60:89-96.

26. Vuong AM, Yolton K, Webster GM, Sjodin A, Calafat AM, Braun JM, Dietrich KN, Lanphear BP, Chen A. Prenatal polybrominated diphenyl ether and perfluoroalkyl substance exposures and executive function in school-age children. Environ Res. 2016;147:556-64.

27. Goudarzi H, Araki A, Itoh S, Sasaki S, Miyashita C, Mitsui T, Nakazawa H, Nonomura K, Kishi R. The Association of Prenatal Exposure to Perfluorinated chemicals with glucocorticoid and androgenic hormones in cord blood samples: the Hokkaido study. Environ Health Perspect. 2017;125(1):111-8.

28. Fei C, McLaughlin JK, Lipworth L, Olsen J. Prenatal exposure to perfluorooctanoate (PFOA) and perfluorooctanesulfonate (PFOS) and maternally reported developmental milestones in infancy. Environ Health Perspect. 2008;116(10):1391-5.

29. Bjerregaard-Olesen C, Bossi R, Liew Z, Long M, Bech BH, Olsen J, Henriksen TB, Berg V, Nost TH, Zhang JJ, et al. Maternal serum concentrations of perfluoroalkyl acids in five international birth cohorts. Int J Hyg Environ Health. 2017:220(2 Pt A):86-93.

30. Lu Z, Song L, Zhao Z, Ma Y, Wang J, Yang H, Ma H, Cai M, Codling G, Ebinghaus $\mathrm{R}$, et al. Occurrence and trends in concentrations of perfluoroalkyl substances (PFASs) in surface waters of eastern China. Chemosphere. 2015;119:820-7.

31. Forns J, Iszatt N, White RA, Mandal S, Sabaredzovic A, Lamoree M, Thomsen C, Haug LS, Stigum H, Eggesbo M. Perfluoroalkyl substances measured in breast milk and child neuropsychological development in a Norwegian birth cohort study. Environ Int. 2015:83:176-82.
32. Chen MH, Ha EH, Liao HF, Jeng SF, Su YN, Wen TW, Lien GW, Chen CY, Hsieh WS, Chen PC. Perfluorinated compound levels in cord blood and neurodevelopment at 2 years of age. Epidemiology (Cambridge, Mass). 2013:24(6):800-8.

33. Jeddy Z, Hartman TJ, Taylor EV, Poteete C, Kordas K. Prenatal concentrations of Perfluoroalkyl substances and early communication development in British girls. Early Hum Dev. 2017;109:15-20.

34. Johansson N, Eriksson P, Viberg H. Neonatal exposure to PFOS and PFOA in mice results in changes in proteins which are important for neuronal growth and synaptogenesis in the developing brain. Toxicol Sci. 2009; 108(2):412-8.

35. Vested A, Ramlau-Hansen CH, Olsen SF, Bonde JP, Kristensen SL, Halldorsson TI, Becher G, Haug LS, Ernst EH, Toft G. Associations of in utero exposure to perfluorinated alkyl acids with human semen quality and reproductive hormones in adult men. Environ Health Perspect. 2013;121(4):453-8.

36. Thibodeaux JR, Hanson RG, Rogers JM, Grey BE, Barbee BD, Richards JH, Butenhoff JL, Stevenson LA, Lau C. Exposure to perfluorooctane sulfonate during pregnancy in rat and mouse. I: maternal and prenatal evaluations. Toxicol Sci. 2003;74(2):369-81.

37. Ji K, Kim S, Kho Y, Paek D, Sakong J, Ha J, Kim S, Choi K. Serum concentrations of major perfluorinated compounds among the general population in Korea: dietary sources and potential impact on thyroid hormones. Environ Int. 2012;45:78-85.

38. Berg V, Nost TH, Hansen S, Elverland A, Veyhe AS, Jorde R, Odland JO, Sandanger TM. Assessing the relationship between perfluoroalkyl substances, thyroid hormones and binding proteins in pregnant women; a longitudinal mixed effects approach. Environ Int. 2015;77:63-9.

39. Wang Y, Rogan WJ, Chen PC, Lien GW, Chen HY, Tseng YC, Longnecker MP, Wang SL. Association between maternal serum perfluoroalkyl substances during pregnancy and maternal and cord thyroid hormones: Taiwan maternal and infant cohort study. Environ Health Perspect. 2014;122(5):529-34.

40. Itoh S, Araki A, Mitsui T, Miyashita C, Goudarzi H, Sasaki S, Cho K, Nakazawa H, Iwasaki Y, Shinohara N, et al. Association of perfluoroalkyl substances exposure in utero with reproductive hormone levels in cord blood in the Hokkaido study on environment and Children's health. Environ Int. 2016:94:51-9.

41. Byrne SC, Miller P, Seguinot-Medina S, Waghiyi V, Buck CL, von Hippel FA, Carpenter DO. Exposure to perfluoroalkyl substances and associations with serum thyroid hormones in a remote population of Alaska natives. Environ Res. 2018;166:537-43.

42. Quach J, Hiscock H, Canterford L, Wake M. Outcomes of child sleep problems over the school-transition period: Australian population longitudinal study. Pediatrics. 2009;123(5):1287-92.

43. Bridgett DJ, Burt NM, Laake LM, Oddi KB. Maternal self-regulation, relationship adjustment, and home chaos: contributions to infant negative emotionality. Infant Behav Dev. 2013;36(4):534-47.

\section{Publisher's Note}

Springer Nature remains neutral with regard to jurisdictional claims in published maps and institutional affiliations.

Ready to submit your research? Choose BMC and benefit from:

- fast, convenient online submission

- thorough peer review by experienced researchers in your field

- rapid publication on acceptance

- support for research data, including large and complex data types

- gold Open Access which fosters wider collaboration and increased citations

- maximum visibility for your research: over $100 \mathrm{M}$ website views per year

At BMC, research is always in progress.

Learn more biomedcentral.com/submissions 